\title{
The Social Reach: 8-Month-Olds Reach for Unobtainable Objects in the Presence of Another Person
}

Psychological Science 2016, Vol. 27(9) 1278-1285 () The Author(s) 2016 Reprints and permissions: sagepub.com/journalsPermissions.nav DOI: $10.1177 / 0956797616659938$ pss.sagepub.com

\author{
Verónica C. Ramenzoni ${ }^{1,2}$ and Ulf Liszkowski ${ }^{1,3}$ \\ ${ }^{1}$ Max Planck Institute for Psycholinguistics, Nijmegen, The Netherlands; ${ }^{2}$ National Scientific \\ and Technical Research Council, Buenos Aires, Argentina; and ${ }^{3}$ Department of Developmental \\ Psychology, University of Hamburg
}

\begin{abstract}
Linguistic communication builds on prelinguistic communicative gestures, but the ontogenetic origins and complexities of these prelinguistic gestures are not well known. The current study tested whether 8-month-olds, who do not yet point communicatively, use instrumental actions for communicative purposes. In two experiments, infants reached for objects when another person was present and when no one else was present; the distance to the objects was varied. When alone, the infants reached for objects within their action boundaries and refrained from reaching for objects out of their action boundaries; thus, they knew about their individual action efficiency. However, when a parent (Experiment 1) or a less familiar person (Experiment 2) sat next to them, the infants selectively increased their reaching for out-of-reach objects. The findings reveal that before they communicate explicitly through pointing gestures, infants use instrumental actions with the apparent expectation that a partner will adopt and complete their goals.
\end{abstract}

\section{Keywords}

social cognition, infant communication, social interaction, cognitive development

Received 1/5/16; Revision accepted 6/27/16

Developmental researchers have long pointed to the extralinguistic foundations of human communication (e.g., Bates, 1979; Bruner, 1975; Werner \& Kaplan, 1963). Across a range of culturally diverse settings, infants begin to point with the canonical index-finger gesture sometime between 9 to 14 months of age (Liszkowski, Brown, Callaghan, Takada, \& de Vos, 2012). This gesture is clearly distinguishable from instrumental-action schemes, such as reaching, because it is often directed at items out of reach, and the conventional hand shape is not effective in grabbing or manipulating those items. Accordingly, most caregivers treat infants' pointing as communicative, typically by commenting on or assisting in the activities in which infants are engaged when they point (e.g., Leung \& Rheingold, 1981; Murphy, 1978). Developmental process-oriented accounts, however, suggest that infants point initially without communicative intent and socialcognitive expectations, which are thought to arise later as a consequence of caregivers' reactions to infants' pointing (e.g., Bates, Camaioni, \& Volterra, 1975; Vygotsky,
1978; for a recent version, see Carpendale \& Carpendale, 2010).

More cognitively oriented accounts have challenged this view of early incompetence. Experimental evidence shows that 12-month-olds point to communicate with others in meaningful ways that entail social-cognitive and cooperative expectations that extend to theory-of-mind and helping scenarios (Liszkowski, 2013). The ontogenetic origins of these skills, however, have remained underspecified. Compatibility between accounts of initial incompetence and those of early cognitive complexity might be found by testing younger infants before they begin to point. If 1-year-olds' pointing is indeed as complex as cognitive accounts claim, and if communicative

\footnotetext{
Corresponding Author:

Verónica C. Ramenzoni, Consejo Nacional de Investigaciones Cientificas y Tecnicas, José Andrés Pacheco de Melo 1854, Buenos Aires C1033AAJ, Argentina

E-mail:vramenzoni@gmail.com
} 
complexities indeed build up gradually from simpler forms as developmental-process accounts suggest, then forms of interactional skills and social expectations should be evident earlier, in the first year of life.

Research on the development of perception and action shows a relation between motor behaviors and the social context within which they occur (Adolph, Tamis-LeMonda, Ishak, Karasik, \& Lobo, 2008; He, Walle, \& Campos, 2015; Karasik, Tamis-LeMonda, Adolph, \& Dimitropoulou, 2008; Tamis-LeMonda et al., 2008). This raises the possibility that before they begin to point, young infants use instrumental actions differently when they are in a social context than when they are alone. Manual reaching is a test case. Object-directed instrumental hand actions emerge around the age of 4 months and very rapidly increase in range and precision. Between the ages of 6 and 8 months, infants become proficient at planning and controlling reaching movements toward objects according to the visuospatial properties of the environment (Corbetta, Thelen, \& Johnson, 2000; Fagard \& Pezé, 1997; von Hofsten \& Fazel-Zandy, 1984). Rochat, Goubet, and Senders (1999) tested the physical conditions under which 6-month-old infants reach for an object and found that they will not reach for objects out of their prehensile space. Thus, by 6 months of age, infants know about the boundaries of their body and action space. This finding may appear to be at odds with observations that 8-month-olds reach for out-of-reach objects offered by an adult (e.g., Blake, O'Rourke, \& Borzellino, 1994), but it is possible that in those cases, infants simply anticipate the trajectory of the offered object toward them. An alternative interpretation, however, is that infants' instrumental reaching at this age begins to serve a social goal in addition to the goal of physically obtaining the object; reaches become social reaches, which entail a partner in the instrumental-action plan.

If it could be demonstrated experimentally that by 8 months of age, infants use an instrumental action with the expectation that a person will detect their goal and assist them in attaining it, this would go a long way in bridging the developmental gap between hand actions that at 6 months serve concrete physical goals (e.g., obtaining an object) and action-decontextualized, referential-communicative acts, such as conventional index-finger pointing, that emerge by the age of 12 months. It would call into question the proposal that 12-month-olds' pointing reflects a full-package evolutionary adaptation and instead support developmental process-oriented accounts, which hold that communicative and social-cognitive skills develop gradually through transformations of previous activities and competencies. It would also reveal an evolutionary commonality with great apes, who use instrumental actions to get cooperative keepers to give them items that are out of reach (van der Goot, Tomasello, \& Liszkowski, 2013).
We devised an experimental procedure that manipulated both the physical and the social environment within which reaching occurred. We measured 8-month-olds' reaching behavior toward objects within and outside their reach, while the infants were alone or in the presence of another person. Unlike the procedure used by Rochat et al. (1999) and in many naturalistic observational studies of young infants' reaching, our procedure did not involve presenting the objects in a person's hands. In this way, we excluded the possibility that differences in infants' reaching would reflect expectations about objects being given to them. Instead, the objects appeared on a tabletop at varying distances, and caregivers sat to the side of the infants. According to our social-reach hypothesis, infants of this age have already developed expectations of changing their social environment with their instrumental actions. Therefore, we predicted that they would persist in reaching for objects placed beyond their reaching abilities selectively when their caregiver was present-expecting that he or she would understand their intention and fulfill it. We conducted a second experiment to see if we could replicate our initial findings and also to test whether infants' expectations of assistance would be circumscribed to the most familiar person they were attached to or had experienced help from, or would also extend to a less familiar person, which would exclude narrow associations as an explanation of this selective reaching in a social context.

\section{Experiment 1}

\section{Method}

Participants. Twenty-two 8-month-old infants (13 female; mean age $=7.97$ months, range $=7.4-8.3$ months, $S D=0.18$ months) and their parents participated in the experiment. On the basis of previous work on the development of perception and action, we aimed for a sample size of 20 , which we decided would be sufficient to reveal a robust effect; 2 of the 22 children were excluded from the final sample because of experimenter error at the beginning of data collection. Participants were recruited from a database of parents who expressed interest in participating in research with their child. The infants in this experiment were primarily White and from middle-class families, living in a medium-sized European city or a surrounding town.

Materials. Each infant sat in a child seat attached to a table and was secured at the waist and shoulders by Velcro straps that allowed for free arm movements (see Fig. 1). The table was covered with a black opaque cloth and had a measuring tape attached to one of its sides. Six plastic animals of different colors and a black rake were used. The rake consisted of a stick ( $80 \mathrm{~cm}$ long $\times 3 \mathrm{~cm}$ wide) with a 


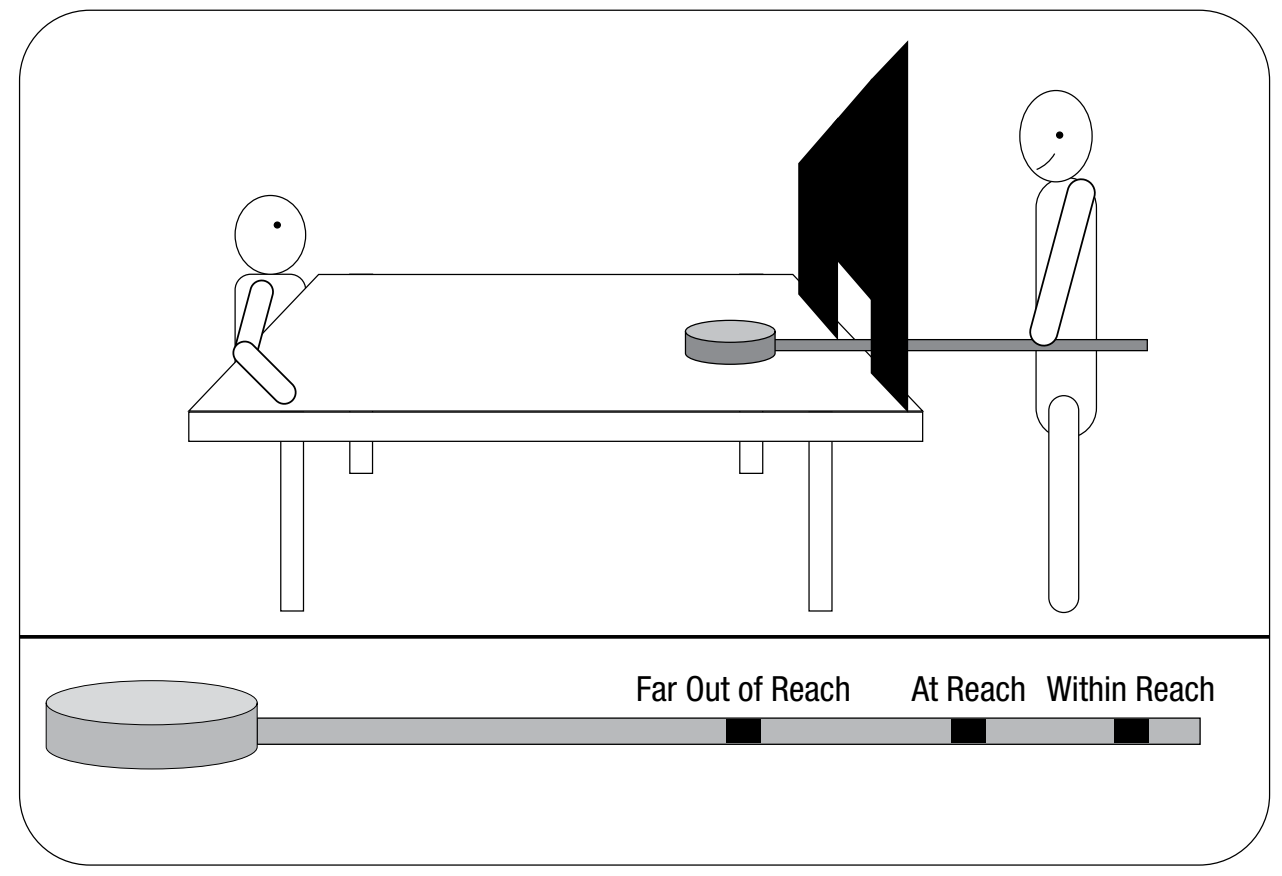

Fig. 1. Illustration of the materials used, the locations of the infant and experimenter during the trials, and the rake with examples of the three distances for placing the objects.

flat circular plate (12 cm in diameter) attached to one of its ends; a measuring tape was attached along the length of the stick, on its underside. A laptop computer was used to calculate the distances from the infants at which to place the objects, and these distances were marked on the rake (these markings were invisible to the infants and were adjusted for each infant). The experimenter hid behind a black occlusion screen $(80 \mathrm{~cm}$ high $\times 50 \mathrm{~cm}$ wide) at the end of the table opposite the infant. In the middle of the bottom portion of the screen, a black cloth covered a small opening through which the rake could be protruded. Two cameras videotaped the infant's behavior from the side and from the top throughout the study. The side-view camera was located to the right of the experimenter and provided a small-screen view that allowed the experimenter to monitor the infant's behavior during each trial.

Procedure. Before the beginning of the experimental session, the experimenter engaged the infant and parent in a short warm-up session to make the infant familiar with the experimenter. Following the warm-up session, the parent was asked to sit the infant in the child seat, and the experimenter helped fasten the security straps. The infant's actual reaching ability was determined by sliding an object from the center of the table along the midline toward the infant. Actual reaching ability was established as the distance at which the infant could encircle the object with his or her fingers.

During the experimental session, the infant was presented with objects at three different distances: far out of reach, at reach, and within reach. Far out of reach was established as the distance $60 \%$ greater than the infant's actual reaching ability, at reach was the distance 30\% greater than the infant's actual reaching ability, and within reach was the distance at $70 \%$ of the infant's actual reaching ability. The average distance at which objects were displayed was $38.77 \mathrm{~cm}(S D=5.70 \mathrm{~cm}$, range $=27-49$ $\mathrm{cm})$ in the far-out-of-reach condition, $30.26 \mathrm{~cm}(S D=$ $6.51 \mathrm{~cm}$, range $=18-40 \mathrm{~cm})$ in the at-reach condition, and $17.02 \mathrm{~cm}(S D=3.19 \mathrm{~cm}$, range $=8-23 \mathrm{~cm})$ in the withinreach condition.

Each infant was presented with two blocks of 6 pseudorandomized trials, for a total of 12 trials. Each of the three distance conditions was presented twice in each block, and a within-reach trial was always presented first to avoid initial frustration. The parent was present (sitting in a chair facing the table, at a $90^{\circ}$ angle from the infant) during one of the blocks and hidden from the infant's view during the other. The order of the blocks was counterbalanced. For the parent-absent condition, the parent hid behind a partition and was allowed to observe the infant through a closed-circuit monitor. In both the parent-absent and the parent-present conditions, the parent was instructed not to respond to the infant's verbal and gestural requests, and was asked to intervene only if the child cried or became fussy.

Before each trial, the experimenter attracted the infant's attention to one of the objects by playing with it above the occlusion screen. The trial started only after the infant had looked for at least $2 \mathrm{~s}$ (cumulatively) at the 
object. The experimenter then hid behind the occlusion screen, placed the object on the rake, and moved it down the table's midline toward the infant, until reaching the desired distance for that trial. The object was then left motionless in that location for a total duration of $8 \mathrm{~s}$. Finally, the experimenter used the rake to withdraw the object behind the screen.

Coding. The infants' behavior was coded using ELAN software (Max Planck Institute for Psycholinguistics, https://tla.mpi.nl/tools/tla-tools/elan/). Reaching, looking, and vocalizing were coded from the videotapes, beginning with the frame when the rake stopped advancing toward the infant and ending with the frame when it began to move back toward the occlusion screen. The coders were blind to the purpose of the study. Both the number and the duration of object-oriented reaching attempts were coded. An object-oriented reaching attempt was defined as either (a) a movement that involved extension of the arm and hand, with the palm down toward the object, with or without grasping motion of the fingers, or (b) a grasping motion with the arm already in extension. Some infants let their arms rest in extension in front of them in between reaching attempts. Cases in which a child reached for the object, let his or her arm rest, and then started moving his or her fingers to try to reach the object were coded as two different reaching attempts. We also coded the number of looks toward the parent or the empty chair and the number of vocalizations while reaching. To check on reliability, we had a second coder randomly select $25 \%$ of the participants from both the parent-present condition and the parent-absent condition and code their behavior. Pearson's $r s$ were as follows: $r(18)=.96, p<.001$, for the average number of reaching attempts; $r(18)=.95, p<.001$, for the average reach duration; $r(18)=.97, p<.001$, for the percentage of trials on which the infant looked toward the parent or empty chair; and $r(18)=.97, p<.001$, for the percentage of trials on which the infant vocalized.

\section{Results}

The infants' reaching ability was on average $25.53 \mathrm{~cm}$ $(S D=3.04 \mathrm{~cm}$, range $=19-32 \mathrm{~cm})$. The number of reaching attempts was similar in the at-reach $(M=2.97, S D=1.96)$ and within-reach $(M=3.1, S D=2.70)$ conditions, because almost all the infants extended their torso forward and leaned into the table in an attempt to obtain the object. The same strategy did not help them succeed when the object was far out of reach. The infants succeeded in pulling the object on $21 \%$ of the trials at the at-reach distance (in $75 \%$ of these trials, the parent was present). When the toy was within reach, the infants were unsuccessful or lost interest in $30 \%$ of the trials.

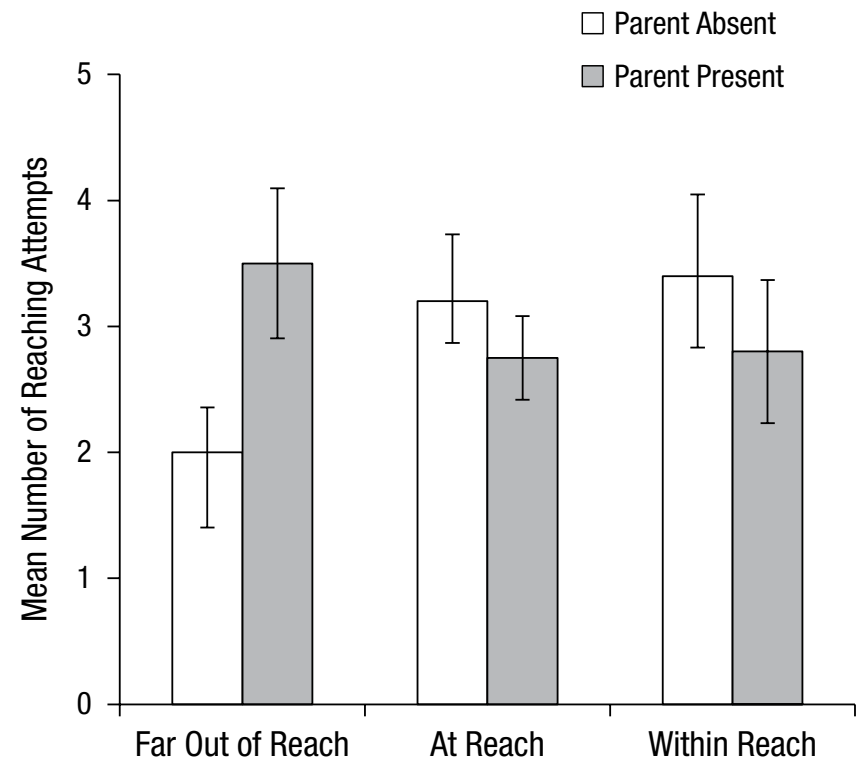

Fig. 2. Results of Experiment 1: mean number of reaching attempts as a function of social condition (parent absent, parent present) and distance (far out of reach, at reach, within reach). Error bars represent $\pm 1 S E M$ for each condition.

We submitted the mean number of reaching attempts to an analysis of variance (ANOVA) with social condition (parent present vs. parent absent) and distance (far out of reach vs. at reach vs. within reach) as factors. This analysis yielded a two-way interaction, $F(2,38)=8.76, p=.01$ (see Fig. 2). A follow-up pairwise comparison revealed that the infants reached significantly more often for the far-out-of-reach objects in the parent-present condition compared with the parent-absent condition, $t(19)=2.45$, $p=.019$. A further comparison confirmed that when alone, the infants tried to obtain the far-out-of reach objects significantly less often than the within-reach objects, $t(19)=6.89, p=.018$.

Overall, 13 of the 20 infants made more reaching attempts toward far-out-of-reach objects when the parent was present than when they were alone. The morphology of reaching attempts (e.g., hand in the air vs. touching the table, presence or absence of grasping motion) did not differ discernibly between conditions. Average duration of reaching attempts also did not differ between conditions-far out of reach: $M=2.0 \mathrm{~s}$ with parent present and $M=1.6 \mathrm{~s}$ with parent absent; at reach: $M=2.0 \mathrm{~s}$ with parent present and $M=2.2 \mathrm{~s}$ with parent absent; within reach: $M=2.0 \mathrm{~s}$ with parent present and $M=1.8 \mathrm{~s}$ with parent absent. The percentage of trials on which the infants vocalized did not differ between the two social conditions (parent-absent trials: 26.47\%; parent-present trials: $30.39 \%), t(19)=0.41, p=.68$. In general, the vocalizations produced were either grumbles of effort when the infants reached for faraway objects, crying and 
moaning when the infants became fussy, and expressions of excitement accompanied by arm waving or banging. Overall, the infants looked more often toward the parent (36.67\% of trials) than toward the empty chair $(25.83 \%$ of trials); however, this difference was not significant, $t(19)=$ $1.26, p=.21$.

\section{Discussion}

When alone, the infants reached for an object that was far out of reach significantly less often than they reached for an object that was within reach. This finding extends previous findings by Rochat et al. (1999), because we controlled for social saliencies (i.e., the objects in the study by Rochat et al. were offered by an adult, whereas the objects in our study were not), and confirms that 8-month-olds calibrate their instrumental reaches to the visuospatial properties of the environment. Our main finding was that when the object was out of reach, the infants selectively increased their reaching attempts in the presence of a potentially helpful caregiver. This finding supports our social-reach hypothesis and provides first evidence that 8-month-old infants use instrumentalaction schemes in expectation that others will detect their goal and assist them in achieving it. Supplementary analyses on vocalizations and looking behavior did not reveal any differences between the parent-present and parentabsent conditions, suggesting that infants' social use of instrumental actions is not yet conventionalized into communicative acts.

One could argue that the infants reached more in the parent-present condition because it was more motivating than the parent-absent condition. However, if this were the case, we would have expected significantly more reaching when the caregiver was present than when the caregiver was absent, regardless of distance. Instead, we found a social effect only for the far-out-of-reach distance. At the same time, the within-reach condition did not yield ceiling effects, which could have precluded a general social facilitation effect. If anything, the infants tended to reach even less in the within-reach condition when the caregiver was present than when the caregiver was absent. Research on the development of perception and action has documented other effects of social facilitation. For example, when walking down slopes that are too steep in the presence of an encouraging person, less experienced infants take more risks, which suggests that the social information outstrips the physical information for these infants (e.g., Adolph et al., 2008). The current setup was rather risk-free, as the infants' torsos were securely supported, and their waists were strapped to the chair. Further, our findings from the parent-absent condition, together with those by Rochat et al. (1999), show that 8-month-olds are rather experienced reachers who know what they can and cannot obtain. Thus, when the 8-month-olds reached for the unobtainable, they did so with the seeming expectation that a person would help them achieve their goal.

It is possible that infants' expectation for help is specific to the caregiver whom they have associated with helping interventions in the past, and perhaps arises as a function of attachment to that caregiver. To test this interpretation, we devised a second experiment in which we replaced the caregiver with a less familiar experimenter. If infants show the social effect also in the presence of a less familiar experimenter, this would make association a less plausible explanation of the effect and would support our hypothesis that infants use their instrumental action with the expectation of eliciting assistance.

\section{Experiment 2}

\section{Method}

Participants. Twenty 8-month-olds (10 female; mean age $=8.02$ months, range $=7.6-8.7$ months, $S D=0.26$ months) and their parents participated in the experiment. Procedures for determining the sample size and for recruitment were identical to those of Experiment 1.

Materials. The materials and apparatus were identical to those of Experiment 1 . The only modification was that a different baby chair had to be used. The chair put the infants' torsos a couple of centimeters further away from the edge of the table than in Experiment 1.

Procedure. The experimental procedure was very similar to that used in Experiment 1, with two main differences. First, the infants performed the task in three social conditions: alone, with the parent present, and with an experimenter present. The only difference between the experimenter-present condition and the parent-present condition was that in the former, a female experimenter, rather than the parent, sat on the chair to the right of the infant. This experimenter had not previously engaged in any extended warm-up periods with the infant, although she had introduced herself to the parent, and the parent typically introduced her to the infant before leaving. In order to prevent the infants from experiencing distress, we always ran the parent-present condition second; the order of the other two conditions was counterbalanced, so that the alone condition was first for half of the infants and the experimenter-present condition was first for the other half. Second, only two distance conditions were tested: far out of reach and within reach $(60 \%$ greater than and 30\% less than maximum reach, respectively). We dropped the middle distance because we wanted to keep the number of trials within the range of infants' 
patience, and because some of the infants in Experiment 1 had succeeded in reaching the at-reach items. The average distance of the objects was $33.25 \mathrm{~cm}(S D=2.52 \mathrm{~cm}$, range $=28-35 \mathrm{~cm}$ ) in the far-out-of-reach condition and $19 \mathrm{~cm}(S D=1.80 \mathrm{~cm}$, range $=16-21 \mathrm{~cm})$ in the withinreach condition. Within each social condition, the infants performed two trials in each distance condition, with the first trial of the within-reach condition always presented first.

Coding. The same coding protocol as in Experiment 1 was followed, except that reaching duration was not coded in this experiment. Correlations between the two coders were as follows: $r(20)=.97, p<.001$, for the average number of reaching attempts in each condition; $r(20)=.98, p<.001$, for the percentage of trials with vocalizations; and $r(20)=.97, p<.001$, for the percentage of trials with looks to the adult or the empty chair.

\section{Results}

The infants' reaching ability was on average $23.75 \mathrm{~cm}$ $(S D=1.89 \mathrm{~cm}$, range $=20-25 \mathrm{~cm})$. The infants succeeded in obtaining the object on $68.57 \%$ of the trials when it was within reach and failed to obtain it or lost interest on the remaining $31.43 \%$ of the trials.

The mean number of reaching attempts produced by each infant was submitted to a within-subjects ANOVA with social condition (parent present vs. alone vs. experimenter present) and distance (far out of reach vs. within reach) as factors. This analysis revealed a two-way interaction between social condition and distance, $F(2,40)=$ $6.12, p=.043$. The infants made more reaching attempts when an adult was present than when they were alone, but only in the far-out-of-reach condition (see Fig. 3) parent-present versus alone condition: $t(21)=-1.74$, $p=.048$; experimenter-present versus alone condition, $t(21)=-2.31, p=.034$.

Overall, 13 of the 20 infants made more reaching attempts toward far-out-of-reach objects when the experimenter was present than when they were alone, and 15 of the 20 infants made more reaching attempts toward far-out-of-reach objects when the parent was present than when they were alone.

Results for the infants' vocalizations showed the same pattern as in Experiment 1. Paired-samples $t$ tests comparing the percentage of trials with vocalizations revealed no significant differences between social conditions (alone trials: 30.1\%; experimenter-present trials: 36.9\%; parent-present trials: $28.3 \%$ ).

The infants did, however, look toward the parent, experimenter, and empty chair more often than in Experiment 1. Their looks were primarily toward the

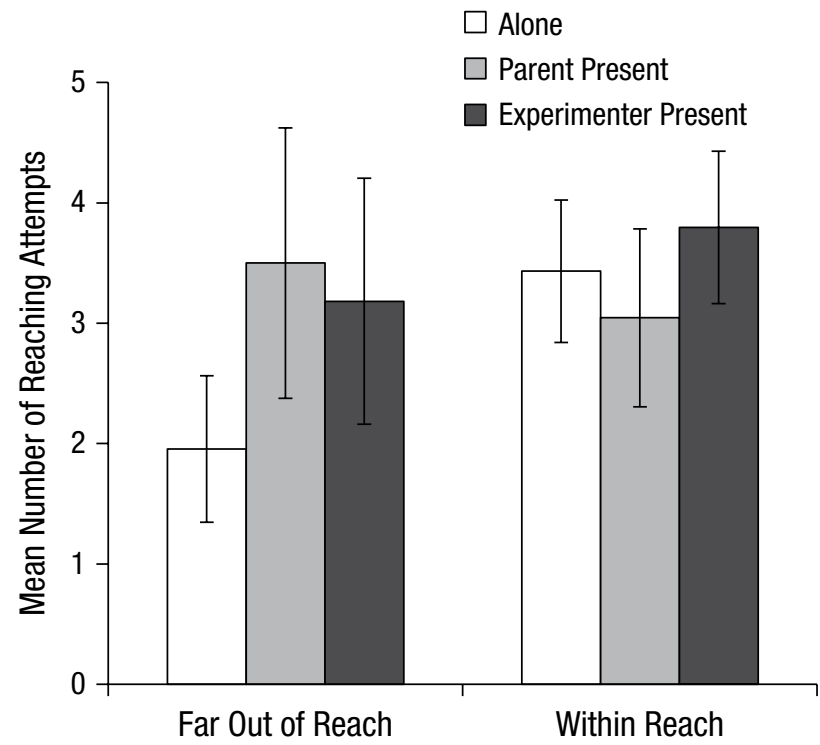

Fig. 3. Results of Experiment 2: mean number of reaching attempts as a function of social condition (alone, parent present, experimenter present) and distance (far out of reach, within reach). Error bars represent $\pm 1 S E M$ for each condition.

experimenter (46.2\% of trials) and the parent $(33.6 \%$ of trials), rather than the empty chair (10.7\% of trials). Pairedsamples $t$ tests showed that the infants looked at the experimenter, $t(21)=4.89, p<.001$, and the parent, $t(21)=3.60, p<.001$, significantly more than at the empty chair. There was no significant difference in the percentage of trials with looks toward the parent and looks toward the experimenter. This pattern suggests that the infants looked to the side in order to check out whether somebody was sitting there and, to an extent, to establish who was sitting next to them.

\section{Discussion}

Experiment 2 replicated the main findings of Experiment 1. When alone, the infants reached for far-out-of-reach items less than for within-reach items (see Fig. 2), and when another person was present, the infants selectively increased their reaching for far-out-of-reach items. As in Experiment 1, the presence of another person did not influence proximal reaching, although there were no ceiling effects, so general social motivation is unlikely to account for our results. The main finding from Experiment 2 is that whether the other person was a caregiver or a less familiar experimenter did not influence the selective social-reaching effect. This finding provides evidence against association-based interpretations. Instead, it reveals that by 8 months of age, infants have a general expectation that another person will cooperate and help them attain the goal of their instrumental actions. The 
subsidiary finding that the infants looked more toward the adult than toward the empty chair is most parsimoniously explained as checking-back behavior (see Liszkowski, Albrecht, Carpenter, \& Tomasello, 2008).

\section{General Discussion}

Our findings provide experimental evidence that young infants reach for unobtainable objects with social-cognitive and cooperative expectations that other persons will understand their goal and assist them in attaining it. The pattern of results across the two experiments excludes several alternative interpretations of what the infants were doing when reaching. The current findings support the cognitively rich interpretation of infant pointing at 12 months (Tomasello, Carpenter, \& Liszkowski, 2007). At the same time, they support developmental process-oriented accounts, which hold that pointing gestures build on earlier-emerging expectations and communicative skills.

Our findings of earlier communicative competence make the emergence of human prelinguistic communication skills at the age of 12 months more traceable. A sudden emergence of communicative and social-cognitive skills is unlikely according to most developmental accounts, and the current findings do not support such a view. In our view, the communicative complexities of 12-month-olds' pointing are a developmental achievement of the first year of life. Although it is clear that longitudinal evidence is needed to support our hypothesis of a relation between infants' earlier interactional competencies and the emergence of pointing, we envision that both cognitive and social processes play a role in such a transition.

Cognitively, to use their actions to communicate, infants would need to understand other individuals as goal-directed agents-and by now, there is plenty of evidence that infants do so before their first birthdays (Wellman, 2010). Infants would also have to understand that a person understands them as goal directed. There is less clear evidence on this point, but when 9-month-old infants request objects, they appear to consider whether a person is or is not willing to help (Behne, Carpenter, Call, \& Tomasello, 2005). These intention-understanding skills are shared with great apes, and great apes are able to communicate in ways that are seemingly similar to those of the 8-months-olds in the current study. However, in contrast to human 8-month-olds, great apes do not transition to more complex forms of gestural communication (van der Goot et al., 2013). Thus, it appears that although an understanding of goals is necessary for communicative pointing, it is not sufficient. Perhaps, then, what is needed, in addition, is social-interactional experience in the form of cooperative helping that builds up expectations of cooperation. Indeed, recent cross-cultural comparisons reveal a predictive positive relation between the amount of triadic interactional experiences and the age at which index-finger pointing begins (Salomo \& Liszkowski, 2013).

Our findings are consistent with the idea that infants initially operate in an irreducible "we-mode" in which they perceive the social context as an extension of their own possibilities of action (Gallotti \& Frith, 2013; see also Werner \& Kaplan's, 1963, "primordial situation"). This proposal shares much with the "shared intentionality" hypothesis (Tomasello, Carpenter, Call, Behne, \& Moll, 2005), although we do not wish to make the claim that infants in their first year of life have a differentiated understanding of shared plans and goals. Rather, this latter understanding may be a later developmental achievement that builds on further social interactional experiences (Butterfill, 2012). Earlier in development, infants may simply assume that other individuals participate in their everyday perceptual and action experiences, such that before they begin to communicate explicitly and conventionally with deictic gestures, they come to expect a helping hand when their own falls short of reaching a goal.

\section{Action Editor}

Brian P. Ackerman served as action editor for this article.

\section{Author Contributions}

V. C. Ramenzoni developed the study concept in collaboration with U. Liszkowski. Both authors contributed to the study design, and V. C. Ramenzoni conceived and built the apparatus used. Testing, data collection, and data coding were performed by research assistants (see Acknowledgments). V. C. Ramenzoni participated in data collection as the second experimenter in Experiment 2. V. C. Ramenzoni performed the data analysis and interpretation under the supervision of U. Liszkowski. Both authors drafted the manuscript, and U. Liszkowski provided critical portions. Both authors approved the final version of the manuscript for submission.

\section{Acknowledgments}

We are grateful to Marloes van der Goot and Mireille Hassemer for help with data collection and coding, and to Marianna Jartó for editing

\section{Declaration of Conflicting Interests}

The authors declared that they had no conflicts of interest with respect to their authorship or the publication of this article.

\section{References}

Adolph, K. E., Tamis-LeMonda, C. S., Ishak, S., Karasik, L. B., \& Lobo, S. A. (2008). Locomotor experience and use of 
social information are posture specific. Developmental Psychology, 44, 1705-1714.

Bates, E. (1979). Intentions, conventions and symbols. In E. Bates, L. Benigni, I. Bretherton, L. Camaioni, \& V. Volterra (Eds.), The emergence of symbols (pp. 69-140). New York, NY: Academic Press.

Bates, E., Camaioni, L., \& Volterra, V. (1975). The acquisition of performatives prior to speech. Merrill-Palmer Quarterly, 21, 205-226.

Behne, T., Carpenter, M., Call, J., \& Tomasello, M. (2005). Unwilling versus unable: Infants' understanding of intentional action. Developmental Psychology, 41, 328-337.

Blake, J., O'Rourke, P., \& Borzellino, G. (1994). Form and function in the development of pointing and reaching gestures. Infant Behavior \& Development, 17, 195-203.

Bruner, J. S. (1975). The ontogenesis of speech acts. Journal of Child Language, 2, 1-19.

Butterfill, S. A. (2012). Interacting mindreaders. Philosophical Studies, 165, 841-863. doi:10.1007/s11098-012-9980-x

Carpendale, J. I., \& Carpendale, A. B. (2010). The development of pointing: From personal directedness to interpersonal direction. Human Development, 53, 110-126. doi: $10.1159 / 000315168$

Corbetta, D., Thelen, E., \& Johnson, K. (2000). Motor constraints on the development of perception-action matching in infant reaching. Infant Behavior \& Development, 23, 351-374.

Fagard, J., \& Pezé, A. (1997). Age changes in interlimb coupling and the development of bimanual coordination. Journal of Motor Behavior, 29, 199-208.

Gallotti, M., \& Frith, C. D. (2013). Social cognition in the wemode. Trends in Cognitive Sciences, 17, 160-165.

He, M., Walle, E. A., \& Campos, J. J. (2015). A cross-national investigation of the relation between infant walking and language development. Infancy, 20, 283-305.

Karasik, L. B., Tamis-LeMonda, C. S., Adolph, K. E., \& Dimitropoulou, K. A. (2008). How mothers encourage and discourage infants' motor actions. Infancy, 13, 366-392.

Leung, E. H., \& Rheingold, H. L. (1981). Development of pointing as a social gesture. Developmental Psychology, 17, 215220.

Liszkowski, U. (2013). Using theory of mind. Child Development Perspectives, 7, 104-109.
Liszkowski, U., Albrecht, K., Carpenter, M., \& Tomasello, M. (2008). Infants' visual and auditory communication when a partner is or is not visually attending. Infant Behavior and Development, 31, 157-167. doi:10.1016/j.infbeh.2007.10.011

Liszkowski, U., Brown, P., Callaghan, T., Takada, A., \& de Vos, C. (2012). A prelinguistic gestural universal of human communication. Cognitive Science, 36, 698-713.

Murphy, C. M. (1978). Pointing in the context of a shared activity. Child Development, 49, 371-380.

Rochat, P., Goubet, N., \& Senders, S. J. (1999). To reach or not to reach? Perception of body effectivities by young infants. Infant and Child Development, 8, 129-148.

Salomo, D., \& Liszkowski, U. (2013). Sociocultural settings influence the emergence of prelinguistic deictic gestures. Child Development, 84, 1296-1307.

Tamis-LeMonda, C. S., Adolph, K. E., Lobo, S. A., Karasik, L. B., Ishak, S., \& Dimitropoulou, K. A. (2008). When infants take mothers' advice: 18-month-olds integrate perceptual and social information to guide motor action. Developmental Psychology, 44, 734-746.

Tomasello, M., Carpenter, M., Call, J., Behne, T., \& Moll, H. (2005). Understanding and sharing intentions: The origins of cultural cognition. Behavioral \& Brain Sciences, 28, 675691.

Tomasello, M., Carpenter, M., \& Liszkowski, U. (2007). A new look at infant pointing. Child Development, 78, 705-722.

van der Goot, M. H., Tomasello, M., \& Liszkowski, U. (2013). Differences in the nonverbal requests of great apes and human infants. Child Development, 85, 444-455.

von Hofsten, C., \& Fazel-Zandy, S. (1984). Development of visually guided hand orientation in reaching. Journal of Experimental Child Psychology, 38, 208-219.

Vygotsky, L. S. (1978). Mind in society: The development of higher mental processes. Cambridge, MA: Harvard University Press.

Wellman, H. M. (2010). Developing a theory of mind. In U. Goswami (Ed.), The Blackwell handbook of cognitive development (2nd ed., pp. 258-284). Oxford, England: Blackwell.

Werner, H., \& Kaplan, B. (1963). Symbol formation: An organismic-developmental approach to language and the expression of thought (3rd ed.). New York, NY: Wiley. 